\title{
An exponential Diophantine equation related to the difference between powers of two consecutive Balancing numbers
}

\author{
Salah Eddine Rihane ${ }^{a}$, Bernadette Faye $^{b}$, \\ Florian Luca ${ }^{c}$, Alain Togbé ${ }^{d}$
}

${ }^{a}$ Université des Sciences et de la Technologie Houari-Boumediène Faculté de Mathématiques, Laboratoire d'Algèbre et Théorie des Nombres Bab-Ezzouar Alger, Algérie salahrihane@hotmail.fr

${ }^{b}$ Department of Mathematics, University Gaston Berger of Saint-Louis Saint-Louis, Senegal bernadette@aims-senegal .org

${ }^{c}$ School of Mathematics, University of the Witwatersrand, Johannesburg, South Africa King Abdulaziz University, Jeddah, Saudi Arabia Department of Mathematics, Faculty of Sciences University of Ostrava, Ostrava, Czech Republic Florian.Luca@wits.ac.za

${ }^{d}$ Department of Mathematics, Statistics, and Computer Science Purdue University Northwest, Westville, USA atogbe@pnw.edu

Submitted: November 6, 2018

Accepted: March 25, 2019

Published online: April 4, 2019

\begin{abstract}
In this paper, we find all solutions of the exponential Diophantine equation $B_{n+1}^{x}-B_{n}^{x}=B_{m}$ in positive integer variables $(m, n, x)$, where $B_{k}$ is the $k$-th term of the Balancing sequence.

Keywords: Balancing numbers, Linear form in logarithms, reduction method. MSC: 11B39, 11J86
\end{abstract}




\section{Introduction}

The first definition of balancing numbers is essentially due to Finkelstein [3], although he called them numerical centers. A positive integer $n$ is called a balancing number if

$$
1+2+\cdots+(n-1)=(n+1)+(n+2)+\cdots+(n+r)
$$

holds for some positive integer $r$. Then $r$ is called the balancer corresponding to the balancing number $n$. For example, 6 and 35 are balancing numbers with balancers 2 and 14, respectively. The $n$-th term of the sequence of balancing numbers is denoted by $B_{n}$. The balancing numbers satisfy the recurrence relation

$$
B_{n}=6 B_{n-1}-B_{n-2}, \text { for all } n \geq 2,
$$

where the initial conditions are $B_{0}=0$ and $B_{1}=1$. Its first terms are

$$
0,1,6,35,204,1189,6930,40391,235416,1372105, \ldots
$$

It is well-known that

$$
B_{n+1}^{2}-B_{n}^{2}=B_{2 n+2}, \text { for any } n \geq 0 .
$$

In particular, this identity tells us that the difference between the square of two consecutive Balancing numbers is still a Balancing number. So, one can ask if this identity can be generalized?

Diophantine equations involving sum or difference of powers of two consecutive members of a given linear recurrent sequence $\left\{U_{n}\right\}_{n \geq 1}$ were also considered in several papers. For example, in [5], Marques and Togbé proved that if $s \geq 1$ an integer such that $F_{m}^{s}+F_{m+1}^{s}$ is a Fibonacci number for all sufficiently large $m$, then $s \in\{1,2\}$. In [4], Luca and Oyono proved that there is no integer $s \geq 3$ such that the sum of $s$ th powers of two consecutive Fibonacci numbers is a Fibonacci number. Later, their result has been extended in [8] to the generalized Fibonacci numbers and recently in [7] to the Pell sequence.

Here, we apply the same argument as in [4] to the Balancing sequence and prove the following:

Theorem 1.1. The only nonnegative integer solutions $(m, n, x)$ of the Diophantine equation

$$
B_{n+1}^{x}-B_{n}^{x}=B_{m}
$$

are $(m, n, x)=(2 n+2, n, 2),(1,0, x),(0, n, 0)$.

Our proof of Theorem 1.1 is mainly based on linear forms in logarithms of algebraic numbers and a reduction algorithm originally introduced by Baker and Davenport in [1]. Here, we will use a version due to Dujella and Pethó in [2, Lemma $5(\mathrm{a})]$. 


\section{Preliminary results}

\subsection{The Balancing sequences}

Let $(\alpha, \beta)=(3+2 \sqrt{2}, 3-2 \sqrt{2})$ be the roots of the characteristic equation $x^{2}-$ $6 x+1=0$ of the Balancing sequence $\left(B_{n}\right)_{n \geq 0}$. The Binet formula for $B_{n}$ is

$$
B_{n}=\frac{\alpha^{n}-\beta^{n}}{4 \sqrt{2}}, \quad \text { for all } n \geq 0 .
$$

This implies that the inequality

$$
\alpha^{n-2} \leq B_{n} \leq \alpha^{n-1}
$$

holds for all positive integers $n$. It is easy to prove that

$$
\frac{B_{n}}{B_{n+1}} \leq \frac{5}{29}
$$

holds, for any $n \geq 2$.

\subsection{Linear forms in logarithms}

For any non-zero algebraic number $\gamma$ of degree $d$ over $\mathbb{Q}$, whose minimal polynomial over $\mathbb{Z}$ is $a \prod_{i=1}^{d}\left(X-\gamma^{(i)}\right)$, we denote by

$$
h(\gamma)=\frac{1}{d}\left(\log |a|+\sum_{i=1}^{d} \log \max \left(1,\left|\gamma^{(i)}\right|\right)\right)
$$

the usual absolute logarithmic height of $\gamma$.

With this notation, Matveev proved the following theorem (see [6]).

Theorem 2.1. Let $\gamma_{1}, \ldots, \gamma_{s}$ be real algebraic numbers and let $b_{1}, \ldots, b_{s}$ be nonzero rational integer numbers. Let $D$ be the degree of the number field $\mathbb{Q}\left(\gamma_{1}, \ldots, \gamma_{s}\right)$ over $\mathbb{Q}$ and let $A_{j}$ be positive real numbers satisfying

$$
A_{j}=\max \left\{D h\left(\gamma_{j}\right),\left|\log \gamma_{j}\right|, 0.16\right\}, \quad \text { for } \quad j=1, \ldots, s .
$$

Assume that

$$
B \geq \max \left\{\left|b_{1}\right|, \ldots,\left|b_{s}\right|\right\} .
$$

If $\gamma_{1}^{b_{1}} \cdots \gamma_{s}^{b_{s}}-1 \neq 0$, then

$$
\left|\gamma_{1}^{b_{1}} \cdots \gamma_{s}^{b_{s}}-1\right| \geq \exp \left(-1.4 \cdot 30^{s+3} \cdot s^{4.5} \cdot D^{2}(1+\log D)(1+\log B) A_{1} \cdots A_{s}\right) .
$$




\subsection{Reduction algorithm}

Lemma 2.2. Let $M$ be a positive integer, let $p / q$ be a convergent of the continued fraction expansion of the irrational $\gamma$ such that $q>6 M$, and let $A, B, \mu$ be some real numbers with $A>0$ and $B>1$. Let

$$
\varepsilon=\|\mu q\|-M \cdot\|\gamma q\|
$$

where $\|\cdot\|$ denotes the distance from the nearest integer. If $\varepsilon>0$, then there is no solution of the inequality

$$
0<m \gamma-n+\mu<A B^{-k}
$$

in positive integers $m, n$ and $k$ with

$$
m \leq M \quad \text { and } \quad k \geq \frac{\log (A q / \varepsilon)}{\log B} .
$$

\section{The proof of Theorem 1.1}

\subsection{An inequality for $x$ versus $m$ and $n$}

The case $n x=0$ is trivial so we assume that $n \geq 1$ and that $x \geq 1$. Observe that since $B_{n}<B_{n+1}-B_{n}<B_{n+1}$, the Diophantine equation (1.1) has no solution when $x=1$.

When $n=1$, we get $B_{m}=6^{x}-1$. In this case, we have that $m$ is odd. Thus, using the Binet formula (2.1), we obtained the following factorization

$$
6^{x}=B_{m}+1=B_{m}+B_{1}=B_{(m+1) / 2} C_{(m-1) / 2},
$$

where $\left\{C_{m}\right\}_{m \geq 1}$ is the Lucas Balancing sequence given by the recurrence $C_{m}=$ $6 C_{m-1}-C_{m-2}$ with initial conditions $C_{0}=2, C_{1}=6$. The Binet formula of the Lucas Balancing sequence is given by $C_{n}=\alpha^{n}+\beta^{n}$. This shows that the largest prime factor of $B_{(m+1) / 2}$ is 3 and by Carmichael's Primitive Divisor Theorem we conclude that $(m+1) / 2 \leq 12$, so $m \leq 23$. Now, one checks all such $m$ and gets no additional solution with $n=1$.

So, we can assume that $n \geq 2$ and $x \geq 3$. Therefore, we have

$$
B_{m}=B_{n+1}^{x}-B_{n}^{x} \geq B_{3}^{3}-B_{1}^{3}=215,
$$

which implies that $m>4$. Here, we use the same argument from [4] to bound $x$ in terms of $m$ and $n$. Since most of the details are similar, we only sketch the argument.

Using inequality (2.2), we get

$$
\alpha^{m-1}>B_{m}=B_{n+1}^{x}-B_{n}^{x} \geq B_{n}^{x}>\alpha^{(n-2) x}
$$


and

$$
\alpha^{m-2}<B_{m}=B_{n+1}^{x}-B_{n}^{x}<B_{n+1}^{x}<\alpha^{n x}
$$

Thus, we have

$$
(n-2) x+1<m<n x+2 .
$$

Estimate (3.1) is essential for our purpose.

Now, we rewrite equation (1.1) as

$$
\frac{\alpha^{m}}{4 \sqrt{2}}-B_{n+1}^{x}=-B_{n}^{x}+\frac{\beta^{m}}{4 \sqrt{2}} .
$$

Dividing both sides of equation (3.2) by $B_{n+1}^{x}$, taking absolute value and using the inequality $(2.3)$, we obtain

$$
\left|\alpha^{m}(4 \sqrt{2})^{-1} B_{n+1}^{-x}-1\right|<2\left(\frac{B_{n}}{B_{n+1}}\right)^{x}<\frac{2}{5.8^{x}} .
$$

Put

$$
\Lambda_{1}:=\alpha^{m}(4 \sqrt{2})^{-1} B_{n+1}^{-x}-1 .
$$

If $\Lambda_{1}=0$, we get $\alpha^{m}=4 \sqrt{2} B_{n+1}^{x}$. Thus $\alpha^{2 m} \in \mathbb{Z}$, which is false for all positive integers $m$, therefore $\Lambda_{1} \neq 0$.

At this point, we will use Matveev's theorem to get a lower bound for $\Lambda_{1}$. We set $s:=3$ and we take

$$
\gamma_{1}:=\alpha, \quad \gamma_{2}:=4 \sqrt{2}, \quad \gamma_{3}:=B_{n+1}, \quad b_{1}:=m, \quad b_{2}:=-1, \quad b_{3}:=-x .
$$

Note that $\gamma_{1}, \gamma_{2}, \gamma_{3} \in \mathbb{Q}(\sqrt{2})$, so we can take $D:=2$. Since $h\left(\gamma_{1}\right)=(\log \alpha) / 2$, $h\left(\gamma_{2}\right)=(\log 32) / 2$ and $h\left(\gamma_{3}\right)=\log B_{n+1}<n \log \alpha$, we can take $A_{1}:=\log \alpha, A_{2}:=$ $\log 32$ and $A_{3}:=2 n \log \alpha$. Finally, inequality (3.1) implies that $m>(n-2) x \geq x$, thus we can take $B:=m$. We also have $B:=m \leq n x+2<(n+2) x$. Hence, Matveev's theorem implies that

$$
\begin{aligned}
\log \left|\Lambda_{1}\right| & \geq-1.4 \times 30^{6} \times 3^{4.5} \times 2^{2} \times(1+\log 2)(\log \alpha)(\log 32)(2 n \log \alpha)(1+\log m) \\
& \geq-2.1 \times 10^{13} n(1+\log m) .
\end{aligned}
$$

The inequalities (3.3), (3.4) and (3.5) give that

$$
x<1.2 \times 10^{13} n(1+\log m)<2.1 \times 10^{13} n \log m,
$$

where we used the fact that $1+\log m<1.7 \log m$, for all $m \geq 5$. Together with the fact that $m<(n+2) x$, we get that

$$
x<2.1 \times 10^{13} n \log ((n+2) x) .
$$




\subsection{Small values of $n$}

Next, we treat the cases when $n \in[2,37]$. In this case,

$$
x<2.1 \times 10^{13} n \log ((n+2) x)<7.8 \times 10^{14} \log (46 x)
$$

so $x<4 \times 10^{16}$.

Now, we take another look at $\Lambda_{1}$ given by expression (3.4). Put

$$
\Gamma_{1}:=m \log \alpha-\log (4 \sqrt{2})-x \log B_{n+1} .
$$

Thus, $\Lambda_{1}=e^{\Gamma_{1}}-1$. One sees that the right-hand side of (3.2) is a number in the interval $\left[-B_{n}^{x},-B_{n}^{x}+1\right]$. In particular, $\Lambda_{1}$ is negative, which implies that $\Gamma_{1}$ is negative. Thus,

so

$$
0<-\Gamma_{1}<\frac{2}{5.8^{x}}
$$

$$
0<x\left(\frac{\log B_{n+1}}{\log \alpha}\right)-m+\left(\frac{\log (4 \sqrt{2}}{\log \alpha}\right)<\frac{2}{5.8^{x} \log \alpha} .
$$

For us, inequality (3.6) is

$$
0<x \gamma-m+\mu<A B^{-x}
$$

where

$$
\gamma:=\frac{\log B_{n+1}}{\log \alpha}, \quad \mu=\frac{\log (4 \sqrt{2})}{\log \alpha}, \quad A=\frac{2}{\log \alpha}, \quad B=5.8 .
$$

We take $M:=4 \times 10^{16}$.

The program was developed in PARI/GP running with 200 digits. For the computations, if the first convergent such that $q>6 M$ does not satisfy the condition $\varepsilon>0$, then we use the next convergent until we find the one that satisfies the condition. In one minute all the computations were done. In all cases, we obtained $x \leq 77$. A computer search with Maple revealed in less than one minute that there are no solutions to the equation (1.1) in the range $n \in[3,37]$ and $x \in[3,77]$.

\subsection{An upper bound on $x$ in terms of $n$}

From now on, we assume that $n \geq 38$. Recall from the previous section that

$$
x<2.1 \times 10^{13} n \log ((n+2) x) .
$$

Next, we give an upper bound on $x$ depending only on $n$. If

$$
x \leq n+2,
$$

then we are through. Otherwise, that is if $n+2<x$, we then have

$$
x<2.1 \times 10^{13} n \log x^{2}=4.2 \times 10^{13} n \log x,
$$


which can be rewritten as

$$
\frac{x}{\log x}<4.2 \times 10^{13} n
$$

Using the fact that, for all $A \geq 3$

$$
\frac{x}{\log x}<A \quad \text { yields } \quad x<2 A \log A
$$

and the fact that $\log \left(4.2 \times 10^{13} n\right)<10 \log n$ holds for all $n \geq 38$, we get that

$$
\begin{aligned}
x & <2\left(4.2 \times 10^{13} n\right) \log \left(\left(4.2 \times 10^{13} n\right)\right. \\
& <8.4 \times 10^{13} n(10 \log n) \\
& <8.4 \times 10^{14} n \log n .
\end{aligned}
$$

From (3.8) and (3.10), we conclude that the inequality

$$
x<8.4 \times 10^{14} n \log n
$$

holds.

\subsection{An absolute upper bound on $x$}

Let us look at the element

$$
y:=\frac{x}{\alpha^{2 n}} .
$$

The above inequality (3.11) implies that

$$
y<\frac{8.4 \times 10^{14} n \log n}{\alpha^{2 n}}<\frac{1}{\alpha^{n}},
$$

where the last inequality holds for any $n \geq 23$. In particular, $y<\alpha^{-38}<10^{-31}$. We now write

$$
B_{n}^{x}=\frac{\alpha^{n x}}{32^{x / 2}}\left(1-\frac{1}{\alpha^{2 n}}\right)^{x}
$$

and

$$
B_{n+1}^{x}=\frac{\alpha^{(n+1) x}}{32^{x / 2}}\left(1-\frac{1}{\alpha^{2(n+1)}}\right)^{x} .
$$

We have

$$
0<\left(1-\frac{1}{\alpha^{2 n}}\right)<e^{y}<1+2 y,
$$

because $y<10^{-31}$ is very small. The same inequality holds if we replace $n$ by $n+1$. Hence, we have that

$$
\max \left\{\left|B_{n}^{x}-\frac{\alpha^{n x}}{32^{x / 2}}\right|,\left|B_{n+1}^{x}-\frac{\alpha^{(n+1) x}}{32^{x / 2}}\right|\right\}<\frac{2 y \alpha^{(n+1) x}}{32^{x / 2}} .
$$


We now return to our equation (1.1) and rewrite it as

$$
\begin{aligned}
\frac{\alpha^{m}-\beta^{m}}{4 \sqrt{2}} & =B_{m}=B_{n+1}^{x}-B_{n}^{x} \\
& =\frac{\alpha^{(n+1) x}}{32^{x / 2}}-\frac{\alpha^{n x}}{32^{x / 2}}+\left(B_{n+1}^{x}-\frac{\alpha^{(n+1) x}}{32^{x / 2}}\right)-\left(B_{n}^{x}-\frac{\alpha^{n x}}{32^{x / 2}}\right),
\end{aligned}
$$

or

$$
\begin{aligned}
\left|\frac{\alpha^{m}}{32^{1 / 2}}-\frac{\alpha^{n x}}{32^{x / 2}}\left(\alpha^{x}-1\right)\right| & =\left|\frac{\beta^{m}}{32^{1 / 2}}+\left(B_{n+1}^{x}-\frac{\alpha^{(n+1) x}}{32^{x / 2}}\right)-\left(B_{n}^{x}-\frac{\alpha^{n x}}{32^{x / 2}}\right)\right| \\
& <\frac{1}{\alpha^{m}}+\left|B_{n+1}^{x}-\frac{\alpha^{(n+1) x}}{32^{x / 2}}\right|+\left|B_{n}^{x}-\frac{\alpha^{n x}}{32^{x / 2}}\right| \\
& <\frac{1}{\alpha^{m}}+2 y\left(\frac{\alpha^{n x}\left(1+\alpha^{x}\right)}{32^{x / 2}}\right) .
\end{aligned}
$$

Thus, multiplying both sides by $\alpha^{-(n+1) x} 32^{x / 2}$, we obtain that

$$
\begin{aligned}
\left|\alpha^{m-(n+1) x} 32^{(x-1) / 2}-\left(1-\alpha^{-x}\right)\right| & <\frac{32^{x / 2}}{\alpha^{m+(n+1) x}}+2 y\left(1+\alpha^{-x}\right) \\
& <\frac{1}{2 \alpha^{n}}+\frac{396 y}{197}<\frac{3}{\alpha^{n}}
\end{aligned}
$$

where we used the fact that $32^{x / 2} /\left(\alpha^{(n+1) x}\right) \leq\left(4 \sqrt{2} / \alpha^{38}\right)^{x}<1 / 2, m \geq(n-2) x \geq$ $n$ and $\alpha^{x} \geq \alpha^{3}>197$, as well as inequality (3.12). Hence, we conclude that

$$
\left|\alpha^{m-(n+1) x} 32^{(x-1) / 2}-1\right|<\frac{1}{\alpha^{x}}+\frac{3}{\alpha^{n}} \leq \frac{4}{\alpha^{l}},
$$

where $l:=\min \{n, x\}$. We now set

$$
\Lambda_{2}:=\alpha^{m-(n+1) x} 32^{(x-1) / 2}-1
$$

and observe that $\Lambda_{2} \neq 0$. Indeed, for if $\Lambda_{2}=0$, then $\alpha^{2((n+1) x-m)}=32^{x-1} \in \mathbb{Z}$ which is possible only when $(n+1) x=m$. But if this were so, then we would get $0=\Lambda_{2}=32^{(x-1) / 2}-1$, which leads to the conclusion that $x=1$, which is not possible. Hence, $\Lambda_{2} \neq 0$. Next, let us notice that since $x \geq 3$ and $m \geq 38$, we have that

$$
\left|\Lambda_{2}\right| \leq \frac{1}{\alpha^{3}}+\frac{1}{\alpha^{38}}<\frac{1}{2}
$$

so that $\alpha^{m-(n+1) x} 32^{(x-1) / 2} \in[1 / 2,3 / 2]$. In particular,

$$
(n+1) x-m<\frac{1}{\log \alpha}\left(\frac{(x-1) \log 32}{2}+\log 2\right)<x\left(\frac{\log 32}{2 \log \alpha}\right)<x
$$

and

$$
(n+1) x-m>\frac{1}{\log \alpha}\left(\frac{(x-1) \log 32}{2}-\log 2\right)>0.9 x-1.4>0 .
$$


We lower bound the left-hand side of inequality (3.15) using again Matveev's theorem. We take

$$
\begin{gathered}
s:=2, \gamma_{1}:=\alpha, \gamma_{2}:=4 \sqrt{2}, b_{1}:=m-(n+1) x, b_{2}:=x-1, \\
D:=2, A_{1}:=\log \alpha, A_{2}:=\log 32, \text { and } B:=x .
\end{gathered}
$$

We thus get that

$$
\log \left|\Lambda_{2}\right|>-1.4 \times 30^{5} \times 2^{4.5} \times 2^{2}(1+\log 2)(\log \alpha)(\log 32)(1+\log x) .
$$

The inequalities (3.14) and (3.19) give

$$
l<4 \times 10^{10} \log x .
$$

Treating separately the case $l=x$ and the case $l=n$, following the argument in [4] we have that the upper bound

$$
x<7 \times 10^{28}
$$

always holds.

\subsection{Reducing the bound on $x$}

Next, we take

$$
\Gamma_{2}:=(x-1) \log (4 \sqrt{2})-((n+1) x-m) \log \alpha .
$$

Observe that $\Lambda_{2}=e^{\Gamma_{2}}-1$, where $\Lambda_{2}$ is given by (3.15). Since $\left|\Lambda_{2}\right|<\frac{1}{2}$, we have that $e^{\left|\Gamma_{2}\right|}<2$. Hence,

$$
\left|\Gamma_{2}\right| \leq e^{\left|\Gamma_{2}\right|}\left|e^{\Gamma_{2}}-1\right|<2\left|\Lambda_{2}\right|<\frac{2}{\alpha^{x}}+\frac{6}{\alpha^{n}} .
$$

This leads to

$$
\left|\frac{\log (4 \sqrt{2})}{\log \alpha}-\frac{(n+1) x-m}{x-1}\right|<\frac{1}{(x-1) \log \alpha}\left(\frac{2}{\alpha^{x}}+\frac{6}{\alpha^{n}}\right) .
$$

Assume next that $x>100$. Then $\alpha^{x}>\alpha^{100}>10^{33}>10^{4} x$. Hence, we get that

$$
\frac{1}{(x-1) \log \alpha}\left(\frac{2}{\alpha^{x}}+\frac{6}{\alpha^{n}}\right)<\frac{8}{x(x-1) 10^{4} \log \alpha}<\frac{1}{2200(x-1)^{2}} .
$$

Estimates (3.20) and (3.21) lead to

$$
\left|\frac{\log (4 \sqrt{2})}{\log \alpha}-\frac{(n+1) x-m}{x-1}\right|<\frac{1}{2200(x-1)^{2}} .
$$


By a criterion of Legendre, inequality (3.22) implies that the rational number $((n+$ $1) x-m) /(x-1)$ is a convergent to $\gamma:=\log (4 \sqrt{2}) / \log \alpha$. Let

$$
\left[a_{0}, a_{1}, a_{2}, a_{3}, a_{4}, a_{5}, a_{6}, \ldots\right]=[0,1,57,1,234,2,1, \ldots]
$$

be the continued fraction of $\gamma$, and let $p_{k} / q_{k}$ be it's $k$ th convergent. Assume that $((n+1) x-m) /(x-1)=p_{k} / q_{k}$ for some $k$. Then, $x-1=d q_{k}$ for some positive integer $d$, which in fact is the greatest common divisor of $(n+1) x-m$ and $x-1$. We have the inequality

$$
q_{54}>7 \times 10^{28}>x-1 .
$$

Thus, $k \in\{0, \ldots, 53\}$. Furthermore, $a_{k} \leq 234$ for all $k=0,1, \ldots, 53$. From the known properties of the continued fraction, we have that

$$
\left|\gamma-\frac{(n+1) x-m}{x-1}\right|=\left|\gamma-\frac{p_{k}}{q_{k}}\right|>\frac{1}{\left(a_{k}+2\right) q_{k}^{2}} \geq \frac{d^{2}}{236(x-1)^{2}} \geq \frac{1}{236(x-1)^{2}},
$$

which contradicts inequality (3.22). Hence, $x \leq 100$.

\subsection{The final step}

To finish, we go back to inequality (3.13) and rewrite it as

$$
\left|\alpha^{m-(n+1) x} 32^{(x-1) / 2}\left(1-\alpha^{-x}\right)^{-1}-1\right|<\frac{3}{\alpha^{n}\left(1-\alpha^{-x}\right)}<\frac{4}{\alpha^{n}} .
$$

Recall that $x \in[3,100]$ and from inequalities (3.17) and (3.18), we have that

$$
0.9 x-1.4<(n+1) x-m<x .
$$

Put $t:=(n+1) x-m$. We computed all the numbers $\left|\alpha^{-t} 32^{(x-1) / 2}\left(1+\alpha^{-x}\right)^{-1}-1\right|$ for all $x \in[3,100]$ and all $t \in[\lfloor 0.9 x-1.4\rfloor,\lfloor x\rfloor]$. None of them ended up being zero and the smallest of these numbers is $>10^{-1}$. Thus, $1 / 10<3 / \alpha^{n}$, or $\alpha^{n}<30$, so $n \leq 3$ which is false.

Acknowledgements. We thank the referee for comments which improved the quality of this manuscript.

F. L. was supported in part by grant CPRR160325161141 and an A-rated scientist award both from the NRF of South Africa and by grant no. 17-02804S of the Czech Granting Agency.

B. F. worked on this paper during her visit to Purdue University Northwest, USA. She thanks the institution for the hospitality. She was also partially supported by a grant from the Simons Foundation.

A. T. was supported in part by Purdue University Northwest. 


\section{References}

[1] A. Baker, H. Davenport: The equations $3 x^{2}-2=y^{2}$ and $8 x^{2}-7=z^{2}$, The Quarterly Journal of Mathematics 20.1 (1969), pp. 129-137, DOI: 10.1093/qmath/20.1.129.

[2] A. Dujella, A. Ретнô: A generalization of a theorem of Baker and Davenport, Quart. J. Math. Oxford Ser. (2) 49.195 (1998), pp. 291-306, DOI: 10.1093/qmathj/49.3.291.

[3] R. P. Finkelstein: The house problem, American Math. Monthly 72 (1965), pp. 1082-1088, DOI: $10.2307 / 2315953$.

[4] F. Luca, R. Oyono: An exponential Diophantine equation related to powers of two consecutive Fibonacci numbers, Proc. Japan Acad. Ser. A 87 (2011), pp. 45-50, DOI: 10.3792/pjaa. 87.45 .

[5] D. Marques, A. Togbé: On the sum of powers of two consecutive Fibonacci numbers, Proc. Japan Acad. Ser. A 86 (2010), pp. 174-176, DoI: 10.3792/pjaa.86.174.

[6] E. M. Matveev: An explicit lower bound for a homogeneous rational linear form in the logarithms of algebraic numbers, II, Izv. Math. 64.6 (2000), pp. 1217-1269, DOI: 10.1070 / im2000v064n06abeh000314.

[7] S. E. Rihane, B. Faye, F. Luca, A. Togbé: On the exponential Diophantine equation $P_{n}^{x}+P_{n+1}^{x}=P_{m}$, To appear in Turkish Journal of Mathematics.

[8] C. A. G. Ruiz, F. LucA: An exponential Diophantine equation related to the sum of powers of two consecutive k-generalized Fibonacci numbers, Coll. Math. 137.2 (2014), pp. 171-188, DOI: $10.4064 / \mathrm{cm} 137-2-3$. 